\title{
A Necessária Regulamentação dos Procedimentos de Substituição Valvar por Via Percutânea pelas Sociedades Médicas
}

\author{
Áurea J. Chaves
}

$\grave{A}$ $\mathrm{s}$ vésperas da reunião do Food and Drug Administration (FDA), que acontecerá neste mês de julho, para avaliar o requerimento para a aprovação pré-comercial nos Estados Unidos da prótese Edwards-SAPIEN ${ }^{\text {TM }}$ (Edwards Lifesciences, Irvine, Estados Unidos) para o tratamento percutâneo da estenose aórtica, duas sociedades médicas - American College of Cardiology (ACC) Foundation e Society of Thoracic Surgeons (STS) - publicaram documento de consenso que menciona pontos fundamentais que devem ser observados antes que procedimentos de substituição valvar por via percutânea estejam largamente disponíveis naquele país. ${ }^{1} \mathrm{O}$ documento enfatiza a necessidade de uma abordagem multidisciplinar dos casos por meio do heart team, define quais são os requisitos para operadores, instalações e protocolos de atendimento, bem como estabelece a necessidade de documentação a longo prazo dos resultados obtidos.

Com preocupação semelhante, a Sociedade Brasileira de Hemodinâmica e Cardiologia Intervencionista (SBHCI) publica, nesta edição da Revista Brasileira de Cardiologia Invasiva (RBCI), seu Consenso de Especialistas sobre o Implante por Cateter de Biopróteses Valvares para o Tratamento da Estenose Aórtica de Alto Risco Cirúrgico, que discorre acerca das recomendações para o procedimento, dos critérios para seleção de pacientes, das implicações para o treinamento e a capacitação dos intervencionistas, da observação dos princípios da ética biomédica, e da conveniência da adoção desse procedimento como medida integrante do rol de políticas públicas de saúde. Foi criado adicionalmente um registro nacional de implante percutâneo de valva aórtica, cujo desenho está publicado por Brito Jr et al., também nesta edição, que tem o intuito de monitorar a incorporação da nova tecnologia e os resultados do procedimento em nosso meio.

Além desse assunto, que domina as discussões da especialidade na atualidade, esta edição traz artigos de muito interesse no cenário do tratamento percutâneo da doença coronária. Collet et al., do Instituto Dante Pazzanese de Cardiologia (São Paulo, SP), apresentam os resultados de pacientes com lesões em pontes de safena, tratados percutaneamente. Trata-se de uma das populações mais desafiadoras dentro do espectro da doença arterial coronária, na qual os resultados da intervenção coronária percutânea (ICP) estão caracteristicamente associados a maior risco de infarto agudo do miocárdio (IAM) periprocedimento, em decorrência da embolização de debris, e em que o maior calibre dos vasos atenua os benefícios dos stents farmacológicos a longo prazo. O conhecimento acumulado na atualidade mostra que a utilização de dispositivos de proteção distal, a menor manipulação do vaso nas fases pré e pós-implante do stent, e o uso de farmacologia adjunta apropriada reduzem as complicações agudas do procedimento e a evolução tardia é marcada pela progressão da doença aterosclerótica nos segmentos não-tratados. Em editorial correspondente, Ellis, da Cleveland Clinic (Cleveland, Estados Unidos), e Ribeiro, do Instituto do Coração do Hospital das Clínicas da Faculdade de Medicina da Universidade de São Paulo (InCor/HCFMUSP - São Paulo, $\mathrm{SP})$, comentam a escassez de dados relativos a esse subgrupo na literatura, os resultados heterogêneos da ICP encontrados, os mecanismos únicos do acometimento aterosclerótico nos enxertos venosos, e os resultados do maior e mais importante estudo randomizado nesse tópico, o ISAR-CABG, apresentado em New Orleans (Estados Unidos), em abril de 2011, no último congresso do American College of Cardiology.

Em outro artigo, Matte et al., do Hospital de Clínicas de Porto Alegre (Porto Alegre, RS), descrevem o panorama nacional da ICP no IAM com supradesnivelamento do segmento ST nos últimos cinco anos, obtido do Registro CENIC. Comparam os resultados de mais de 20 mil pacientes, coletados em 252 centros, em 22 Estados, de acordo com as regiões do Brasil, com enfoque na ICP primária e nos tratamentos adjuntos farmacológico e mecânico. Mattos, do Instituto Dante Pazzanese de Cardiologia, profundo conhecedor do assunto por sua atuação de mais de uma década no Registro, exalta em seu editorial as diversas conquistas alcançadas ao longo da história, mas aponta três características que são os maiores desafios a ser enfrentados para que a CENIC alcance consistência científica maior: o caráter voluntário do registro, a falta de adjudicação dos resultados e o curto período de obtenção dos 
dados, restrito à fase hospitalar. Aponta, no final, medidas de impacto para renovar esse projeto, que é um dos pilares científicos da SBHCl.

Dando sequência a uma série de estudos que avaliam o primeiro stent brasileiro, publicada na $\mathbf{R B C I}$, Takimura et al., do InCor (São Paulo, SP), trazem os resultados experimentais da avaliação por tomografia de coerência óptica (TCO) da versão farmacológica desse stent, com eluição abluminal de sirolimus. O stent, denominado Inspiron ${ }^{\mathrm{TM}}$ (Scitech Produtos Médicos Ltda., Goiânia, GO), foi comparado ao stent BioMatrix ${ }^{\mathrm{TM}}$ (Biosensors International, Cingapura), com eluição também abluminal de biolimus A9, em coronárias porcinas, demonstrando equivalência de resultados em termos de inibição da hiperplasia intimal, segundo os dados obtidos pelos autores. Chamié e Attizzani, no momento atuando no Harrington-McLaughlin Heart \& Vascular Institute (Cleveland, Estados Unidos), uma das instituições de grande destaque no desenvolvimento de projetos com a TCO, fazem minuciosa revisão das possibilidades do método, que oferece oportunidade única para avaliação, in vivo, da interação stent-vaso e caminha, segundo os autores, para se tornar a modalidade de imagem padrão na avaliação da resposta vascular ao implante de stents. Pedem cautela na interpretação dos resultados desse estudo experimental, já que, em sua visão, o método não foi aplicado em sua plenitude. Trata-se de um editorial imperdível para os leitores em geral e, em especial, para os interessados em métodos de imagem.
Finalizando, gostaria de chamar sua atenção para a excelência dos demais artigos originais publicados, que exploram temas como a evolução a longo prazo de pacientes com lesão de tronco de coronária esquerda não-protegido tratados por meio de stents farmacológicos; dois artigos provenientes do Registro InCor, que avaliam a evolução tardia de enxertos de safena tratados com stents farmacológicos e o efeito protetor da circulação colateral no IAM complicado por choque cardiogênico tratado por ICP primária; o desempenho tardio dos stents com liberação de everolimus em lesões coronárias de novo; o impacto do sexo feminino nos resultados hospitalares da ICP contemporânea; a validação de novo protocolo que simplifica a supervisão dos dispositivos de compressão radial para obtenção de hemostasia; a relação custo-efetividade do dispositivo de oclusão vascular comparado à compressão mecânica da via de acesso femoral; e a experiência com o sistema de reversão de fluxo durante angioplastia carotídea.

Aproveitem!

\section{Áurea J. Chaves} Editora

\section{REFERÊNCIA}

1. Holmes DR, Mack MJ. Transcatheter valve therapy: a Professional Society Overview from the American College of Cardiology Foundation and the Society of Thoracic Surgeons. J Am Coll Cardiol [Internet]. 2011 Jun 21 [cited 2011 Jun 26];58(4). Available from: http://content.onlinejacc.org/cgi/reprint/58/ 4/445.pdf 